\title{
El acompañamiento tutorial en escritura académica universitaria como práctica intercultural
}

\author{
Tutoring in Academic Writing \\ as an Intercultural Practice
}

\author{
Karla Susana Lombardi González \\ Instituto Tecnológico y de Estudios Superiores de Occidente (iteso), México \\ klombardi@iteso.mx \\ Juan Carlos Silas Casillas
}

Instituto Tecnológico y de Estudios Superiores de Occidente (iteso), México silasjc@iteso.mx

8

\section{RESUMEN}

Este artículo reporta una experiencia de acompañamiento tutorial en escritura académica con estudiantes indígenas en una universidad privada en el occidente de México. Los resultados superaron las expectativas y fueron influidos por dos factores básicos: 1) el rescate de la horizontalidad como práctica relacional y 2) propiciar que los estudiantes vinculen los conceptos académicos con su experiencia personal e históricamente situada en lo concreto. Se recomienda a las universidades explorar este tipo de intervenciones para favorecer la interculturalidad estructural y el desarrollo académico de sus estudiantes indígenas.

Palabras clave: Tutoría; educación superior; intercultural; escritura.

\begin{abstract}
This article reports a tutorial experience in academic writing with indigenous students at a private university in Western Mexico. The results were better than expected and were influenced by two basic factors: the rescue of horizontality as relational practice and leading students to relate academic concepts with their personal experience and historically situated in the concrete. Universities are encouraged to explore this type of intervention to promote the structural interculturality and the academic development of their indigenous students.
\end{abstract}

Keywords: Tutoring; Higher Education; Intercultural; Writing.

Fecha de recepción: 30/10/2019

Fecha de aceptación: 08/02/2020

El acompañamiento tutorial en escritura académica universitaria como práctica intercultural • 7

Karla Susana Lombardi González y Juan Carlos Silas Casillas. DIDAC 76 (2020): 7-13 


\section{Interculturalidad como concepto guía}

El concepto de interculturalidad ha estado en el discurso público, político y académico desde hace más de cuatro décadas y es objeto de análisis en disciplinas tales como la educación, la psicología, la sociología y hasta la mercadotecnia o la administración. Ha sido más evidente en áreas como la educación intercultural bilingüe o la educación popular, buscando generar cambios desde lo comunitario (Krainer \& Guerra, 2016). El interés de los gobiernos nacionales fue evidente a partir del cambio de siglo, principalmente para atender demandas en torno a la diversidad cultural en las sociedades (Bello, 2009). De alguna manera se puede decir que "como aspiración, la interculturalidad es parte de un proyecto de nación" (Schmelkes, 2013, p. 5).

Hay varias formas de entender la interculturalidad, las cuales se pueden agrupar en dos: la conservadora (Viaña, 2010) y la crítica (Walsh, 2009). La primera procura arraigarse a un contexto monocultural sin tocar las causas de la desigualdad social y cultural que se operacionaliza desde una visión funcional del sistema, en la cual se legitima el orden asimétrico y hegemónico (Walsh, 2009). La segunda, más crítica, reflexiva y emancipadora, implica desarrollar procesos que se inician desde abajo hacia arriba y que buscan la trasformación social, "dirigidos a fortalecer lo propio (...), ampliar los espacios de lucha y de relación con los demás sectores en condiciones de simetría y a impulsar cambios estructurales o sistémicos" (Walsh, 2012, p. 34).

Este trabajo aborda la interculturalidad de forma crítica, desde la visión de Walsh, e incorpora preceptos filosóficos relacionados con el respeto y la promoción de la diversidad; asimismo, reconoce los elementos que históricamente han caracterizado a las culturas originarias y a la universidad como estructura académica.

La interculturalidad (pre)supone:

1. Fortalecer, desde una perspectiva crítica, lo propio, y asume la autoestima basada en la identidad y en los saberes culturales necesarios para las relaciones equitativas, con la intención de crecer según la propia tradición y saber posicionarse entre las distintas culturas desde la autocrítica como un ser colectivo (Walsh, 2012).

2. Establecer una relación de igualdad, procurando que el estudiante indígena borre sus estigmas de diferencia, para transitar a "la postura que sostiene que el otro puede y debe crecer desde lo que es, desde su propia identidad" (Schmelkes, 2013, p. 5).

3. Fomentar una educación que tenga al entorno inmediato de los estudiantes como fundamento, e incorpore elementos y contenidos de horizontes culturales diversos, con el fin de enriquecer el proceso formativo (López $\& \mathrm{Ji}$ ménez, 2001) viendo al otro diferente, no como una amenaza, sino como alguien cuya diferencia le es favorable y cuyo contacto le enriquece como persona (Schmelkes, 2013).

4. Fomentar las negociaciones y los intercambios culturales a través de diversas vías que permitan la construcción de espacios de encuentros personales y culturales (Krainer \& Guerra, 2016), con la intención de proyectar su derecho a la autodeterminación, el derecho de construir un mundo de acuerdo con sus necesidades y puentes hacia otras culturas (Casillas \& Santini, 2006).

5. Asumir a las culturas como vivas y dinámicas, adaptables y promotoras de los cambios a partir del contacto con otras. Respetar visiones y culturas diferentes, formas de vivir y propuestas de solución de los problemas (Schmelkes, 2013).

En este marco, lo trascendente será aprovechar las diferencias y diseñar estrategias para abrir "la posibilidad de impulsar un proceso de complementación y enriquecimiento entre la ciencia moderna y otros saberes; analiza, además, cómo otras tradiciones saben y deciden lo que deben y quieren saber" (Krainer \& Guerra, 2016, p. 16).

En lo tocante a las prácticas de lectura y escritura en la educación superior, Hernández (2016) señala cómo se relacionan con el entorno cultural que

8 - El acompañamiento tutorial en escritura académica universitaria como práctica intercultural Karla Susana Lombardi González y Juan Carlos Silas Casillas. DIDAC 76 (2020): 7-13 
las origina. Su producción y dominio están inextricablemente vinculados con las maneras locales de comunicarse que, a su vez, se anclan en culturas particulares, por lo que será fundamental tomar en cuenta el contexto situado en que se produjeron. La comunicación oral y escrita se liga a prácticas sociales amplias, vinculadas con una dimensión comunitaria y el papel del entorno (Zavala, 2009). Por esto no se puede esperar que la práctica comunicativa de una persona se transforme sin un proceso intencional de mediación. De tal manera que la práctica educativa, cuya pretensión busque desarrollar la escritura académica en un marco de interculturalidad se encuentre, con la necesidad de co-construir significados (Bajour, 2009).

\section{Bosquejo metodológico de acompañamiento e investigación}

La práctica de acompañamiento a universitarios indígenas tuvo lugar durante el año escolar 20182019, en una institución de educación superior (IES) particular en el occidente de México. A pesar de las buenas intenciones declaradas, al tratarse de una universidad urbana con décadas de operación, su estructura no se adapta con facilidad a alumnos culturalmente diferenciados. Los estudiantes indígenas afrontan distintos retos académicos por los elementos personales y relacionales que tienen. Hacen frente a situaciones de aprendizaje donde están implicados los procesos de lectura, escritura y oralidad académica, los cuales son requisitos para el dominio de nuevas estructuras y sistemas lingüísticos que les demandan sus estudios universitarios (Archanco, 2018).

En el semestre de otońo 2018 se puso en marcha un esquema piloto con 10 alumnos indígenas, a partir de sus necesidades expresas. Este esquema inició con un diagnóstico de habilidades de comunicación y atendió tanto lo "urgente" (los trabajos que deben entregar para sus materias) como lo "importante" (las estructuras que deben dominar para un mejor desempeño académico). La característica sobresaliente de esta intervención fue la flexibilidad operativa e intentar acoplar el esquema escolásti- co-comunicativo universitario usual con la riqueza de su legado cultural y la experiencia de ser universitario. La experiencia fue enriquecedora y favoreció la reflexión de los participantes.

Se presenta una reseña desde tres ángulos: 1) la dinámica del acompañamiento al estudiante indígena, 2) las implicaciones de la lectura, escritura y oralidad académicas frente a sus procesos de aprendizaje y a su lengua, y 3) la del alumno indígena, desde lo académico frente a su cultura. Posteriormente se señala cómo se hizo la recuperación, sistematización y análisis de los datos.

El acompañamiento se inició reconociendo que, para el universitario indígena, la construcción del conocimiento académico requiere vincularlo con su contexto histórico. De no ser así, el aprendizaje se construye de manera fragmentada y disociada de su apropiación, discursividad, identidad y significado de su participación social. En este sentido, se requirió entender que la ciencia es tanto un hábito de pensamiento como una forma de vida (Cornejo, 2011) y que, desde la postura crítica, no se contrapone con la cultura del estudiante indígena, sino que es un elemento de diálogo.

La práctica de acompańamiento, desarrollada por la profesora, implicó apartarse de la perspectiva del "déficit", de pensar al indígena como un alumno con carencias que deben subsanarse. Este tipo de posturas regularmente contribuyen a crear o fortalecer preconceptos que se traducen en una especie de paternalismo bondadoso que disminuye las expectativas sobre el desempeño de los muchachos. La postura del acompañamiento se concentró en entender al indígena como un individuo libre en sus decisiones y que reconoce sus necesidades para elegir si solicita o no un acompańamiento. La persona responsable del acompañamiento fue consciente desde el inicio de que el alumno indígena: 1) proviene de condiciones distintas a las de un estudiante urbano-mestizo, 2) que su historia personal está sujeta a tensiones socioeconómicas y una cosmovisión con símbolos culturales y lingüísticos propios, y 3) su lengua materna revela los procesos étnicos y culturales de su comunidad. A partir de esta postura, el 
acompañamiento se orientó a construir relaciones horizontales (en la medida de lo posible), de intercambio entre los saberes culturales del estudiante con los saberes académicos, a través de las dinámicas mediadoras de la lectura, la escritura y la oralidad de su disciplina académica.

Para generar un ambiente de intercambio y de apertura dentro del espacio de acompañamiento, la facilitadora buscó establecer un diálogo informal para romper con los estereotipos del maestro y el alumno en la institución universitaria. Fue significativo observar que durante las primeras sesiones, los alumnos reportaron que percibían tener un nivel académico deficiente y que sus habilidades estaban por debajo de los demás compañeros. Ellos habían solicitado el acompańamiento porque se sentían con menos habilidades y capacidades para enfrentar lo académico. Los procesos de diagnóstico de sus competencias de escritura académica no se basaron en pruebas estandarizadas o calificación de las habilidades de lectura, escritura y oralidad académicas, sino a través de la dinámica del "primer diálogo", donde se les motivó a contar acerca de sí, de su cultura, de sus intereses, de la elección de su carrera y de sus sueños. Esa narración ayudó a la construcción de un ambiente de confianza, una relación cercana que evita la competición y permite el error como detonador de aprendizaje. Esto se basó en la idea de que las instituciones bosquejan configuraciones culturales que reflejan su acción desde el poder académico legitimado y con base en ello entienden y organizan la atención a la diversidad.

La práctica del acompañamiento tutorial evitó en todo momento la visión homogeneizante de un modelo único de acompañamiento, y se propuso al estudiante indígena como un sujeto activo "con una presencia de igualdad diferenciada y con una capacidad de reconocimiento frente a sus formas de aprender" (Herrera, 2006). Fue importante el papel de la facilitadora como apoyo para el estudiante en su (re)construcción como sujeto científico relacionado con su acción cotidiana, a la luz de procesos de lectura y escritura, y como una mediadora sociocultural de la formación académica-científica.
En relación con el esquema metodológico para la obtención de información, éste fue correspondiente a las ideas base del enfoque cualitativo y sirviéndose del método interpretativo básico (Merriam $\&$ asociados, 2002). Dado que las sesiones de acompańamiento se desarrollaron en un salón de clase, se pudo tener tranquilidad, espacio, equipo y mobiliario suficiente para el trabajo individualizado. Para la obtención de información se optó por grabar en su totalidad las sesiones de acompañamiento. Se solicitó la autorización de los estudiantes y se les garantizó el anonimato. Se les aclaró que el foco de interés era específicamente cómo se desarrollaban tanto las sesiones como sus aprendizajes. Se transcribió el contenido de las sesiones, se tomaron fotografías del pizarrón (los ejemplos y temas revisados en la sesión), se conservaron los trabajos de clase sobre los que se desarrolló el acompañamiento y se escribió una "bitácora reflexiva" de cada sesión en la que se plasmaba, además del nombre del estudiante, el tema y la finalidad de cada sesión, los ejercicios realizados. Adicionalmente, se anotaban las incidencias de cada sesión. Este cúmulo de datos y fuentes sirvieron para tener claridad de la utilidad de los ejercicios y las reflexiones de los alumnos.

Se cuenta con los datos referidos de 68 sesiones de acompañamiento tutorial. Con la finalidad de respetar el anonimato comprometido, sólo se dirá que los participantes fueron 10 alumnos de diferentes etnias, todos ellos de primer y segundo semestre, y que ésta se trataba de su primera experiencia de educación universitaria.

\section{Breve recuento y resultados}

El trabajo de acompańamiento con los alumnos se centró en facilitar el desarrollo de habilidades de escritura académica. En ocasiones se incluyeron elementos de lectura de comprensión y oralidad. La principal dificultad inicial de los alumnos fue comprender el contenido de las lecturas académicas para las asignaturas de su área disciplinar. Este obstáculo radicó en su dominio del castellano, en especial por no estar familiarizados con la semántica de los conceptos y la dificultad de asociarlos a sus propios con-

10 - El acompañamiento tutorial en escritura académica universitaria como práctica intercultural Karla Susana Lombardi González y Juan Carlos Silas Casillas. DIDAC 76 (2020): 7-13 
textos cercanos, pero diferenciados de su cultura. Algunos estudiantes señalaban que leían varias veces y no retenían o no entendían.

Para atender este asunto, se propuso a los alumnos que observaran que en su cultura también existía una práctica similar y que a partir de sus saberes tradicionales se podía explicar o relacionar con lo que se revisaba en clase. Esta práctica fue particularmente exitosa en la comprensión del concepto revisado, que a su vez funcionó como catalizador de las frases que querían usar en sus escritos académicos, mismos que se elaboraron de manera paralela.

Algunos ejemplos relevantes son: 1) un estudiante de medios audiovisuales que revisaba el concepto de "hegemonía" lo comprendió en diálogo con lo establecido en su comunidad; 2) un alumno de derecho que buscaba entender textos sobre las formas de organización política y democrática lo logró a través de la revisión de las prácticas dialógicas en su pueblo; 3) uno de ingeniería que intentaba entender un texto de dinámicas de enfriamiento y lo comparó con las prácticas tradicionales en su comunidad de origen; 4) un estudiante de administración batallando con textos que abordaban ideas abstractas sobre sustentabilidad que al momento de poner los conceptos en la concreción de su experiencia familiar, logró asirlos; 5) otro de educación con temas teóricos sobre el aprendizaje que logró "destrabar" su comprensión al relacionarlo con su historia personal escolar.

La experiencia permitió ver cómo el estudiante indígena, al revisar textos académicos (abstractos y esquemáticos por naturaleza), tenía dificultades para relacionar los contenidos específicos de su disciplina con los saberes de su cultura, en especial porque ignoraba intencionalmente lo suyo y suponía que el conocimiento "nuevo y escolástico" era lo relevante en ese momento y no el conocimiento de su tradición. Esto provocaba una disociación y minusvaloración. Los tres elementos (la facilitadora, el alumno indígena y el texto) que participan en la dinámica del acompañamiento, requieren de la concurrencia de lazos culturales, así como el intercambio de formas simbólicas a través de la expresión oral (obviamente usando la propia voz) y escrita con la palabra del texto.

Un elemento clave para el desarrollo de las habilidades de expresión escrita fue una estrategia de tres pasos que consistió en: 1) elaboración de textos en solitario (el alumno), 2) revisión en conjunto de las ideas y la expresión de las mismas (facilitadora y alumno) y 3) reelaboración en conjunto buscando las formas de expresar lo pensado (facilitadora y alumno). El acompañamiento tutorial se puede asumir entonces como un espacio que favorece el diálogo entre los saberes académicos universitarios y la tradición oral de su comunidad de origen. En esta práctica, el alumno narra y pone en perspectiva los saberes de su cultura, para posteriormente buscar la manera de manifestarlos de acuerdo con la tradición académica escrita científica-escolástica. En este sentido, la voz es la marca de la subjetividad en el lenguaje y, para que haya transmisión, éste debe llevar la marca del que transmite (Larrosa, 2008). Con esto en mente, la tarea de la facilitadora se centró en construir un ambiente detonador que permitiera al estudiante percibir cómo sus condiciones culturales son un insumo valioso para resignificar y negociar sus razonamientos, identidad, percepciones y representaciones frente al texto académico.

\section{Conclusiones y recomendaciones}

El proceso de acompańamiento tutorial con estudiantes indígenas universitarios lleva a entender que la interculturalidad es un concepto que no admite asimetrías. Y si las hay, corresponde que los educadores las combatan en modo directo (Krainer \& Guerra, 2016). La experiencia de acompañamiento permite ver la interculturalidad desde lo práctico y detectar que el facilitador es quien debe estar atento para no resaltar "lo que pretende enseñar al otro" (que se asocia con una asimetría casi colonizadora) y será clave su capacidad de escuchar al otro, ese otro diferente, para colaborar en sus conocimientos.

Las prácticas de lectura y escritura, en especial en la educación superior, se vinculan estrechamente con el entorno cultural que las origina y caracteriza. Están inmersas en competencias comunicativas re- 
lacionadas con momentos y culturas particulares, y su estudio y promoción deben considerar el contexto en que se produjeron (Hernández, 2016). Se trata de actividades ligadas a prácticas sociales amplias, vinculadas con una clara dimensión comunitaria y el papel del entorno (Zavala, 2009). Entonces, un elemento que fundamenta la práctica educativa centrada en la promoción de la escritura académica en un marco de interculturalidad es la construcción de significados con otros, sin necesidad de cerrarlos (Bajour, 2009). Esto es una condición fundamental de la escucha y supone una conciencia donde la construcción de sentidos es un acto dialógico y no meramente individual.

El acompañamiento tutorial resalta la trascendencia de favorecer la transmisión de la experiencia de vida indígena y tener una participación dinámica de escucha. Ésta cobra sentido desde la generación de espacios a fin de dar voz a los estudiantes para que construyan significados desde su experiencia (Larrosa, 2008). Al respecto, se debe resaltar que, en la dinámica pedagógica de la práctica tutorial universitaria, el texto científico — a pesar de las rigurosidades académicas que contiene-, cobrará sentido desde la vivencia y las características culturales de quien lo usa.

Cuando el indígena universitario afronta textos académicos, tiene reconfiguraciones simbólicas y significativas (a través de las relaciones intercultura- les) de aprender provenientes de sus tradiciones, y tiene que confrontarse con una cultura académica que está basada en otras tradiciones, en la cual participa el "otro código disciplinar". En este sentido, resulta relevante para la práctica del acompañamiento tutorial que se oriente desde la textualidad, sobre la transmisión de saberes emancipadores, vinculando la historia de vida del indígena y la historia escolar (Meirieu, 2013), ya que los saberes académicos muchas veces se muestran sin significación.

La práctica de acompańamiento tutorial lleva a entender que hay una serie de desafíos en la apertura de las relaciones interculturales en espacios académicos universitarios. Y uno de estos retos es que la misma universidad reconozca la diversidad y las tradiciones indígenas no sólo como culturas ancestrales históricas, sino como parte inclusiva dentro de los procesos de enseñanza-aprendizaje.

Por último, se debe resaltar que la experiencia descrita permite recomendar que se desarrollen trabajos de acompańamiento tutorial cercano a los intereses y las necesidades de los estudiantes indígenas. Estos procesos requieren minuciosidad, seguimiento y una fuerte inversión, ya que será a través del trabajo individualizado, basado en el contexto originario de cada persona y el reconocimiento de las prácticas de la comunidad, donde los alumnos podrán verbalizar sus ideas y con ello "traerlas" al presente contextualizado de la universidad.

REFERENCIAS

Archanco, P. (2018). Sobre la práctica de la lectura en la escuela: supuestos, continuidades y rupturas. Clase 20, bloque 5 . Argentina: FLACso. Recuperado de https://prezi.com/p/ xixonz3d2jlk/sobre-la-practica-de-la-lectura-en-la-escuela-supuestos-continuidades-y-rupturas/

Bajour, C. (2009). Oír entre líneas: el valor de la escucha en las prácticas de lectura. Imaginaria. Revista Quincenal sobre Literatura Infantil y Juvenil, 256. Recuperado de https://imaginaria.com.ar/2009/06/oir-entre-lineas-el-valor-de-laescucha-en-las-practicas-de-lectura/

Bello, Á. (2009). Derechos indígenas y ciudadanías diferenciadas en América Latina y el Caribe. Implicancias para la educación. En L. E. López (Ed.), Interculturalidad, educación y ciudadania (pp. 57-76). La Paz: Funproeib Andes.

Casillas, M. de L. \& Santini, L. (2006). Universidad intercultural. Modelo educativo. México: CGEIB-SEP.
Cornejo, J. (2011). La lectura en la construcción escolar de la ciencia: el relato de una experiencia. Revista Ciencia Escolar Enseñanza y Modelización, 1(1), 27-41. Recuperado de http://www.revistacienciaescolar.cl/articulos/cies-2011-118.pdf

Hernández, G. (2016). Literacidad académica. Ciudad de México: UAM.

Herrera, G. (2006). Encuentro entre la cultura oral y la cultura escrita, una reflexión epistemológica en estudiantes indígenas en la Universidad Pedagógica Nacional. México: ANUIES.

Krainer, A. \& Guerra, M. (2016). Interculturalidady educación. Desafíos docentes. Quito: FLACSO.

Larrosa, J. (2008). Aprender de oído. Intervención en el ciclo de debates Liquidación por Derribo: Leer, Escribir y Pensar en la Universidad, organizado por La Central, en Barcelona.

12 - El acompañamiento tutorial en escritura académica universitaria como práctica intercultural Karla Susana Lombardi González y Juan Carlos Silas Casillas. DIDAC 76 (2020): 7-13 
López, L. \& Jiménez, C. (2001). Educación intercultural. Guatemala: Ministerio de Educación.

Meirieu, P. (2013). La opción de educar y la responsabilidad pedagógica. Conferencia dictada en el Ministerio de Educación de la Nación, en el Palacio Sarmiento, octubre 2013. Recuperado de http://www.educ.ar//recursos/ver? $\mathrm{id}=121626$

Merriam, S. \& asociados (2002). Qualitative Research in Practice. Examples for Discussion and Analysis. Boston: Jossey-Bass.

Rockwell, E. (2007). Huellas del pasado en las culturas escolares. Revista de Antropología Social, 16, 175-212.

Schmelkes, S. (2013). Educación para un México intercultural. Sinéctica, 40, 1-12. Recuperado de http://www.scielo.org. $\mathrm{mx} /$ scielo.php?script $=$ sci_arttext $\&$ pid $=S 1665$ 109X2013000100002\&lng=es\&tlng=es
Viańa, J. (2010). Reconceptualizando la Interculturalidad. En Construyendo interculturalidad crítica. La Paz: Instituto Internacional de Integración del Convenio Andrés Bello.

Walsh, C. (2009). Hacia una comprensión de la interculturalidad. Tukari. Espacio de Comunicación Intercultural, 11, 6-7. Recuperado de http://www.tukari.udg.mx/sites/default/files/11.\%20Interculturalidad\%20y\%20pueblos\%20ind\%C3\%ADgenas.pdf

Walsh, C. (2012). Interculturalidad critica y (de)colonialidad. Ensayos desde Abya Yala. Quito: Abya Yala.

Zavala, V. (2009). La literacidad o lo que la genta hace para leer y escribir. En D. Cassany, Para ser letrados (23-35). Barcelona: Paidós.

Karla Susana Lombardi González. Obtuvo la maestría en Comunicación de la Ciencia y la Cultura por el Instituto Tecnológico y de Estudios Superiores de Occidente (ITESO). Especializada en Políticas Culturales y Gestión Cultural por la Universidad Autónoma Metropolitana (UAM). Licenciada en Ciencias de la Educación con la especialidad en Educación Cognoscitiva en el ITEso. Doctorante en Educación Interinstitucional por la Universidad Iberoamericana de Puebla, Ciudad de México, León y el IтEso. Desde el año 2000 a la fecha es profesora en los departamentos de Lenguas y Psicología, Educación y Salud en el ITEso. En esta misma institución participa en un equipo de investigación interdepartamental con el tema de literacidad académica y estudiantes universitarios indígenas.

Juan Carlos Silas Casillas. Obtuvo el doctorado en Educational Policy and Leadership en la Universidad de Kansas en el año 2000. Cuenta con una maestría en Educación y la licenciatura en Psicología Educativa por la Universidad del Valle de Atemajac (UNIVA), Guadalajara. De 2004 a 2008 se desempeńó como director del Centro Interdisciplinario de Estudios sobre Calidad de la Educación y Superación de la Pobreza (CIECESP) en la Universidad de Monterrey (UDEM). Desde el año 2008 a la fecha es investigador y docente en el Departamento de Psicología, Educación y Salud del Instituto Tecnológico y de Estudios Superiores de Occidente (ITESO). Desde 2016 es coordinador del doctorado interinstitucional en Educación. Es miembro del Sistema Nacional de Investigadores (SNI), nivel 1. Sus temas de investigación y publicaciones se enfocan en el balance público-privado en la educación superior, la relación entre las instituciones educativas y la comunidad, y el pensamiento científico-crítico en alumnos universitarios. 